\title{
The Sauvé-Kapandji Procedure
}

\author{
Alberto Lluch, $\mathrm{MD}, \mathrm{PhD}^{1}$ \\ ${ }^{1}$ Institut Kaplan for Surgery of the Hand and Upper Extremity, \\ Barcelona, Spain \\ J Wrist Surg 2013;2:33-40.
}

\begin{abstract}
Address for correspondence Alberto Lluch, MD, PhD, Institut Kaplan for Surgery of the Hand and Upper Extremity, Paseo Bonanova, 9, 2-2, 08022 Barcelona, Spain (e-mail: albertolluch@institut-kaplan.com).
\end{abstract}

\begin{abstract}
\section{Keywords}

- arthrodesis

- distal radioulnar joint

- ulnocarpal impaction

- triangular fibrocartilage

Arthrodesis is the most reliable and durable surgical procedure for the treatment of a joint disorder, and its only disadvantage is the loss of motion of the fused joint. The distal radioulnar joint can be arthrodesed, while forearm pronation and supination are maintained or even improved by creating a pseudoarthrosis of the ulna just proximal to the arthrodesis. This is known as the Sauvé-Kapandji (S-K) procedure. The SauvéKapandji differs from the Darrach procedure in that it preserves ulnar support of the wrist, as the distal radioulnar ligaments and ulnocarpal ligaments are maintained. Aesthetic appearance is also superior after the $\mathrm{S}-\mathrm{K}$ procedure, as the normal prominence of the ulnar head, most noticeable when the forearm is in pronation, is maintained. However, the S-K is not free of possible complications, such as nonunion or delayed union of the arthrodesis, fibrous or osseous union at the pseudoarthrosis, and painful instability at the proximal ulna stump. All of these complications can be prevented if a careful surgical technique is used.
\end{abstract}

Many procedures have been described for the management of the altered distal radioulnar joint (DRUJ), not only because there is no single procedure superior to another, but mainly because a broad spectrum of pathologies requires different surgical techniques for each lesion. ${ }^{1}$ The most common alterations of the DRUJ are joint incongruencies secondary to deformities of the distal radius from malunited fractures, distal radial epiphysiodesis, or Madelung deformities. Isolated joint instabilities after ruptures of the distal radioulnar ligaments are also common. Primary degenerative arthritis is unusual but may be observed after fractures of the distal radius or ulna. In other cases, the problems may be related to the ulnocarpal joint rather than the DRUJ, as in triangular fibrocartilage complex (TFCC) tears or the so called ulnocarpal impaction syndrome when the ulna is longer than the radius.

Resection of the ulnar head was first described by Malgaine in $1855^{2}$ and later popularized by Darrach in 1912 and $1913 .^{3,4}$ It is an easy procedure to perform with early good functional results, although not free of complications. These consist mainly of instability of the proximal ulna and loss of grip strength. To avoid these complications, several techniques of partial resection of the ulnar head while preserving the ulnar styloid and the ligaments inserting at its base have been described.

Chronic instability of the DRUJ can be treated by varied ligament reconstructive procedures, but all require a long period of postoperative immobilization, and they frequently fail to restore normal joint stability.

In 1921, Baldwin reported that loss of pronation and supination of the forearm, after malunited distal radial fractures, had been successfully treated by excising a $2-\mathrm{cm}$ segment of the ulna proximal to the DRUJ, thus creating a pseudoarthrosis. ${ }^{5}$ In 1936, Sauvé and Kapandji described a similar technique, with the variant that a DRUJ arthrodesis was added to the ulnar pseudoarthrosis. ${ }^{6}$ However, the so called Sauvé-Kapandji technique had already been published in 1931 by Berry ${ }^{7,8}$ after being performed the previous year, using a bone peg instead of a screw or a Kirschner wire (Kwire) to stabilize the DRUJ arthrodesis.

The combination of arthrodesis of the DRUJ and ulnar resection-osteotomy proximal to the arthrodesis, for restoration of pronation and supination of the forearm, has the advantage over ulnar head resection of preserving ulnar support of the wrist, as the distal radioulnar ligaments and ulnocarpal ligaments are preserved. It also allows for
Copyright (c) 2013 by Thieme Medical Publishers, Inc., 333 Seventh Avenue, New York, NY 10001, USA.

Tel: +1(212) 584-4662.
DOI http://dx.doi.org/ $10.1055 / \mathrm{s}-0032-1333465$. ISSN 2163-3916. 
shortening of the ulnar head, which cannot always be done with resection arthroplasties that preserve distal radioulnar ligaments such as the Bowers, Feldon, or Watson procedures. Another advantage of the S-K technique is that the postoperative immobilization is shorter, which is an added benefit for the patient.

\section{Patients and Methods}

The S-K technique can be successfully used for the treatment of any pathology involving the distal radioulnar and ulnocarpal joints. The most common indication is a distal radioulnar instability or incongruency after a distal metaphyseal radius fracture. This technique allows shortening of the ulna for the treatent of an ulnocarpal impaction, as well as recovery of forearm rotation when it is decreased. Simultaneous correction of a severe distal radial angulation can be performed at the same time, but doing so will add a technical difficulty to the procedure. Lengthening of the radius is not necessary, as the length can be restored from a shortening of the ulna. In some distal radial osteotomies, it will not be possible to stabilize the ulnar head to the radius with a screw, and K-wires may have to be used instead, which does not provide stability.

One of the best indications for the S-K technique is ulnocarpal impaction from a long ulna secondary to a fracture of the distal radius that has healed with shortening and minimal angulation. This technique enables correction of the ulnar length while preserving the TFCC and ulnocarpal ligaments.

The S-K technique is also indicated for the treatment of isolated DRUJ instabilities secondary to ligament ruptures. Ligament repair techniques do not always offer adequate joint stability and require long periods of immobilization of the wrist and elbow, which are very uncomfortable and disabling for the patient and, in some cases, lead to a loss of forearm rotation. ${ }^{8}$

Chronic or difficult-to-repair TFCC tears can also be treated with the S-K procedure. The patient can resume normal daily activities after the skin sutures are removed, as the screw provides for stabilization of the DRUJ, thus avoiding long periods of immobilization required after TFCC repairs. When the DRUJ is arthrodesed, there are no strains into the TFCC, and lesions will "spontaneously" repair if their vascularity allows for it. We started treating chronic TFCC tears with the S-K technique after observing spontaneous healing of delayed unions of styloid fractures of the ulna after DRUJ arthrodesis.

This technique is also indicated for the treatment of early synovitis of the DRUJ in rheumatoid patients. ${ }^{9,10}$ After the arthrodesis, joint synovitis will permanently disappear, preventing rupture of the distal radioulnar and ulnocarpal ligaments. When the ulnar head is completely dislocated, the procedure will not provide ligamentous stability, as the ligaments will already have been destroyed, but it will provide axial bone support for the lunate, mainly in cases with ulnar translocation of the carpus. This may be of benefit if a simultaneous radiolunate arthrodesis has been per- formed, as this will offer more bone contact between the lunate and the distal radius and ulna.

Seventy patients who underwent the technique just described were reviewed on an average of 8 years after surgery (from 3 to 12.5 years). Thirty-eight were women and 32 men, with one woman presenting a Madelung deformity underwent surgery in both wrists. The average patient's age was 40 , ranging from 25 to 75 years of age. The indications for surgery were DRUJ instability and/or incongruence after fractures of the distal radius in 44 wrists; primary DRUJ osteoarthritis in 3 wrists; isolated DRUJ instabilities secondary to ligament injury in 10 wrists; rupture of the TFCC in 3; rheumatoid arthritis in 10; and Madelung deformity in 3. A simultaneous corrective osteotomy of the distal radius was performed in 10 wrists: eight that were sequelae after a fracture of the distal radius and two wrists with a Madelung deformity.

\section{Surgical Technique}

The DRUJ is approached dorsally through an inverted V incision centered over the ulnar head (-Figs. 1a-d). The dorsal sensory branch of the ulnar nerve running obliquely from proximal-anterior to distal-dorsal can be identified and protected at the distal part of the incision. The fifth extensor compartment is divided longitudinally, and the extensor digiti minimi tendon is retracted toward the radial side. Doing so provides a better visualization of the dorsal edge of the sigmoid notch of the radius, as well as preventing inadvertent injury to this small-caliber tendon. The dorsal DRUJ capsule is divided longitudinally close to its insertion into the radius, and then removed to enable adequate exposure of the ulnar head and neck. The extensor carpi ulnaris (ECU) tendon glides inside an osteofibrous tunnel dorsal to the styloid process of the ulna, which is independent from the extensor retinaculum. Neither of these structures should be disturbed, as they are important stabilizers of the ECU tendon and the DRUJ. ${ }^{11}$

With the forearm in pronation, the joint cartilage and subchondral bone of the ulnar head facing the surgeon are removed, leaving a flat or slightly convex surface of cancellous bone. The ulnar head is perforated with a $3.2-\mathrm{mm}$ drill, with the entrance at the center of the denuded ulnar head and the exit anterior to the ECU sheath. The direction of the drill should be perpendicular to the long axis of the radius and ulna (-Figs. 2a,b). The triangular skin flap should be retracted to visualize and confirm that the exit of the drill bit is just anterior to the sixth compartment of the extensor retinaculum. With a small knife blade, the puncture wound into the extensor retinaculum produced by the drill should be slightly enlarged longitudinally, to later facilitate easy identification of the screw entrance point.

Next, the head of the ulna is osteotomized just proximal to where the joint cartilage ends ( - Fig. 3 ). This osteotomized head can then be displaced and rotated into supination, allowing visualization of the sigmoid notch of the radius and the proximal ulnar stump ( $\mathbf{- F i g . 4}$ ). The osteotomy can be done with an oscillating saw or with small cutting forceps. If a power saw is used, the blade should be thin with fine teeth, to prevent excessive seeding of bone particles into the soft tissues. 

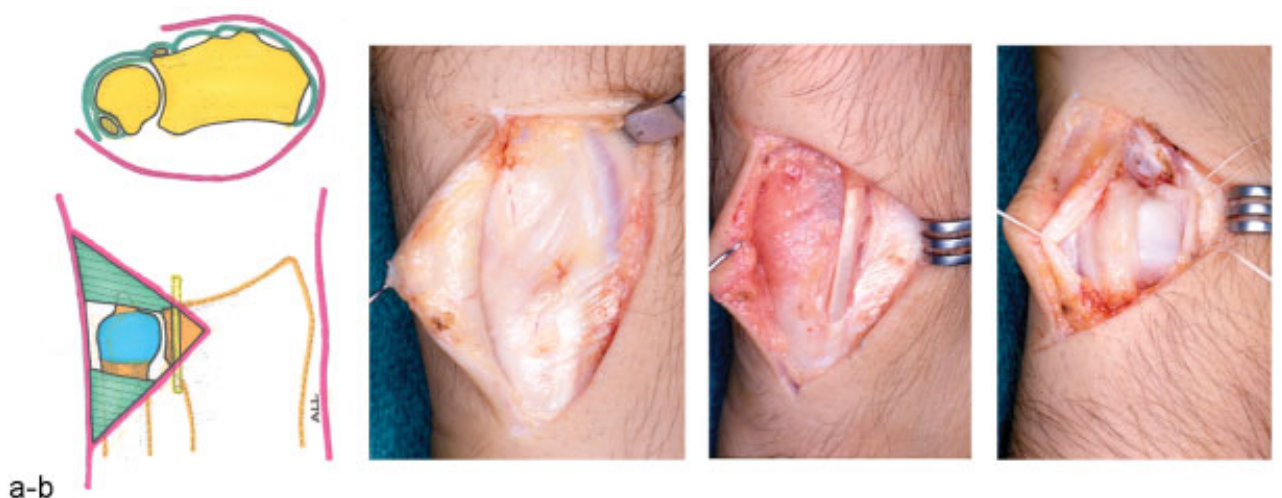

c-d

Fig. 1 (a) (Top) Cross-section anatomy of the DRUJ. The forearm is in pronation, and the ulnar head is covered by the DRUJ capsule and the extensor retinaculum. The fifth extensor compartment stabilizes the extensor digiti minimi tendon at the most ulnar border of the distal radius. The extensor carpi ulnaris (ECU) tendon is stabilized by its own sheath, separate from the extensor retinaculum, lateral to the styloid process of the ulna. (Bottom) Ulnar head after the extensor retinaculum has been ulnarly reflected and the dorsal radioulnar capsule removed. (b) Exposure of the extensor retinaculum. (c) Division of the fifth extensor compartment allows for identification and radial displacement of the extensor digiti minimi tendon. (d) Longitudinal division of the capsule exposes the head of the ulna. Joint synovitis, when present, should be removed as exposure will improve.

Next, a segment of the proximal ulnar stump should be removed. In those cases in which there is no length discrepancy between radius and ulna, only $5 \mathrm{~mm}$ of ulna should be removed. If the ulna is longer than the radius, an additional segment of the ulna, equivalent to the length discrepancy, should be removed. A power saw or preferably a small bone rongeur can be used. Bone excision of the proximal ulna should be done after minimal soft tissue retraction, to avoid damage to the insertions of the pronator quadratus (PQ) muscle and the interosseous membrane (IOM) into the proximal ulna. Retractors should be placed very carefully around the proximal ulna, and all resection should be done looking directly from the space created after the ulnar head has been displaced, while rotating the radius into pronation

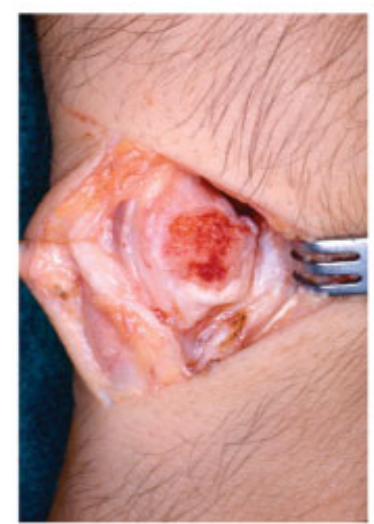

a

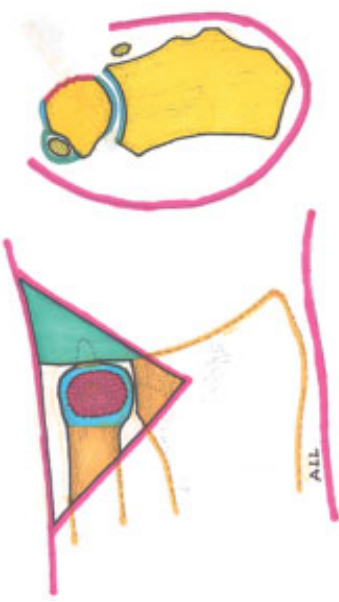

b

Fig. 2 (a) With the forearm in pronation, the joint cartilage and subchondral bone of the ulnar head facing the surgeon are removed, leaving a slightly convex surface of cancellous bone. (b) The ulnar head is perforated using a 3.2-mm drill bit, with the entrance at the center of the denuded ulnar head, which exits anteriorly to the styloid process and ECU sheath. The direction of the drill should be perpendicular to the long axes of the radius and ulna. and supination. After this is done, all bone debris should be removed, as well as the periosteum of the segment of ulna that has already been removed.

The joint cartilage and subchondral bone of the sigmoid notch of the radius are then excised. As the joint surface is slightly concave, it is difficult to bite into it with a bone rongeur. The procedure will be facilitated by starting at the angle formed by the union of the dorsal cortex and the dorsal rim of the sigmoid notch of the radius. Removal of the cartilage and subchondral bone can be continued later with a bone rongeur and a curette.

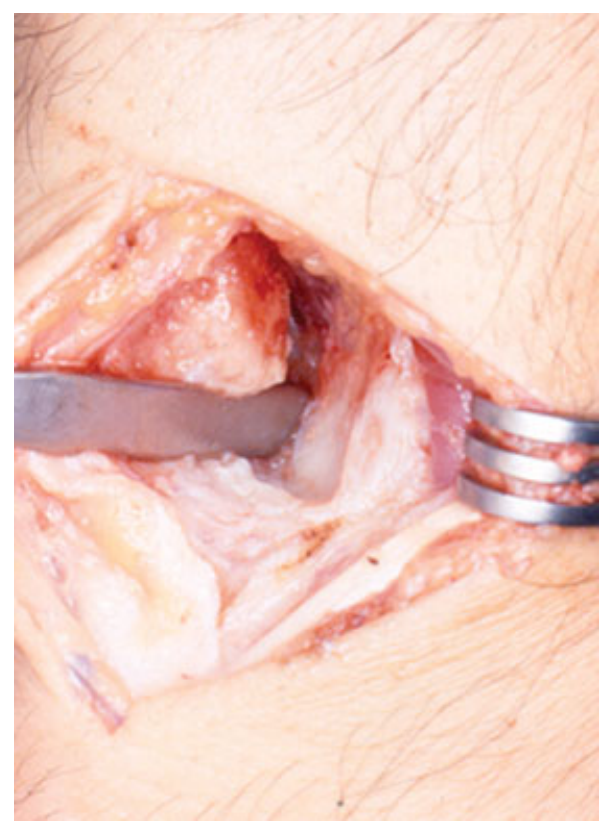

Fig. 3 The head of the ulna is osteotomized at the level of the neck and then displaced and rotated into supination, allowing visualization of the sigmoid notch of the radius. A segment of the proximal ulna is excised, and the joint cartilage and subchondral bone of the sigmoid notch of the radius are removed. 


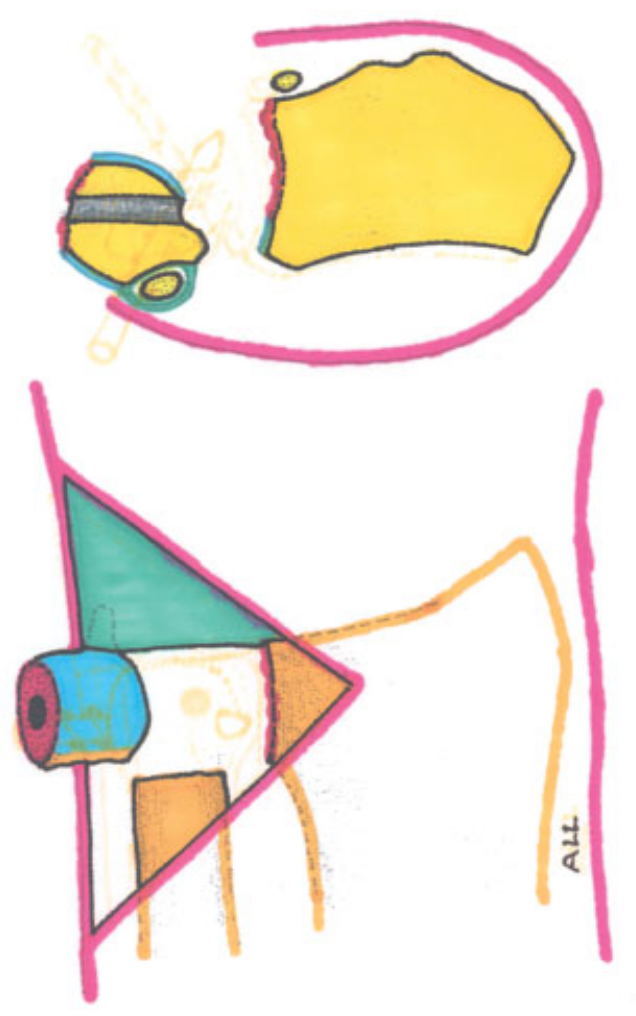

Fig. 4 Schematic drawing of the osteotomy of the head of the ulna done just at the proximal edge of the joint cartilage. Rotation of the head of the ulna into supination allows visualization of the sigmoid notch of the radius.

A malleolar lag screw is inserted perpendicularly into the head of the ulna through the previously made drill hole. The entrance of the screw should be just anterior to the compartment for the ECU tendon, and the screw is introduced until its tip protrudes $\sim 3 \mathrm{~mm}$ from the center of the denuded surface of the head of the ulna (-Figs. $\mathbf{5 a}, \mathbf{b}$ ). Using the screwdriver, the head of the ulna is reduced opposite to the radius until the protruding tip of the screw is placed at the center of the sigmoid notch of the radius. Before inserting the screw into the radius, the surgeon should carefully check the correct position of the head of the ulna in relation to the radius. The most distal part of the ulnar head should be $\sim 2 \mathrm{~mm}$ shorter than the ulnar edge of the distal radius. A slightly shorter ulnar head will prevent ulnocarpal impingement and damage to the TFCC, as well as placing the ulnocarpal ligaments under slight tension. The distal edge of radial sigmoid joint may be difficult to visualize, as it is covered by the radial insertion of the TFCC. As it is very important not to arthrodese the ulna head in a position longer than the radius, it may be necessary to detach the dorsal radioulnar ligament partially for a more precise identification of the distal radioulnar joint. The selftapping malleolar screw is introduced through the distal end of the radius, perpendicular to the long axis of the bone, without the need of drilling. The screw should be from 35 to $40 \mathrm{~mm}$ in length, depending on the size of the wrist, and it
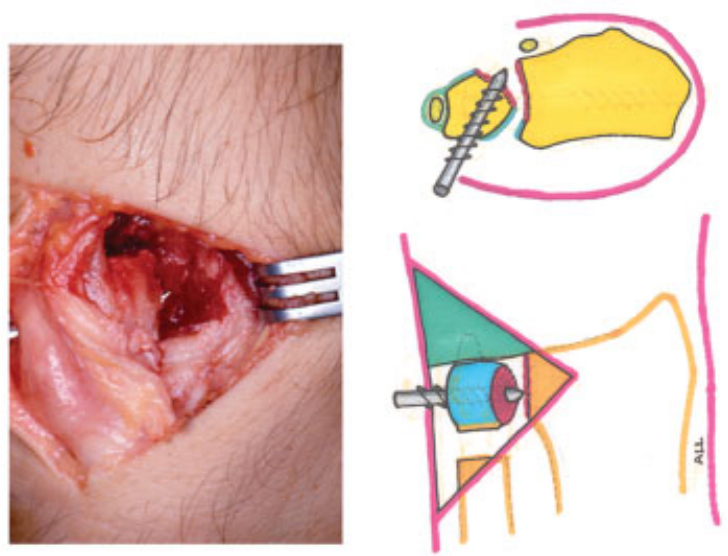

a

b

Fig. 5 (a) A malleolar screw is inserted perpendicular to the head of the ulna through the previously made drill hole. The screw should enter just anterior to the compartment of the ECU tendon, and be introduced until its tip protrudes $\sim 3 \mathrm{~mm}$ from the center of the denuded surface of the head of the ulna. (b) Schematic drawing of the previous intraoperative photograph.

should not protrude through the opposite radial cortex, as this may cause injury from friction to the abductor pollicis longus tendon. The screw is advanced into the radius until the bone surfaces to be arthrodesed have been moderately compressed and the head of the screw is slightly introduced into the head of the ulna (-Figs. $\mathbf{6 a , b}$ ).

The wrist is immobilized with a circular plaster bandage from 7 to 10 days. After the bandage and the skin sutures are removed, the wrist is left free, and the patient is instructed to bring the forearm into maximal pronation and supination. The patient should not be continuously rotating the forearm,
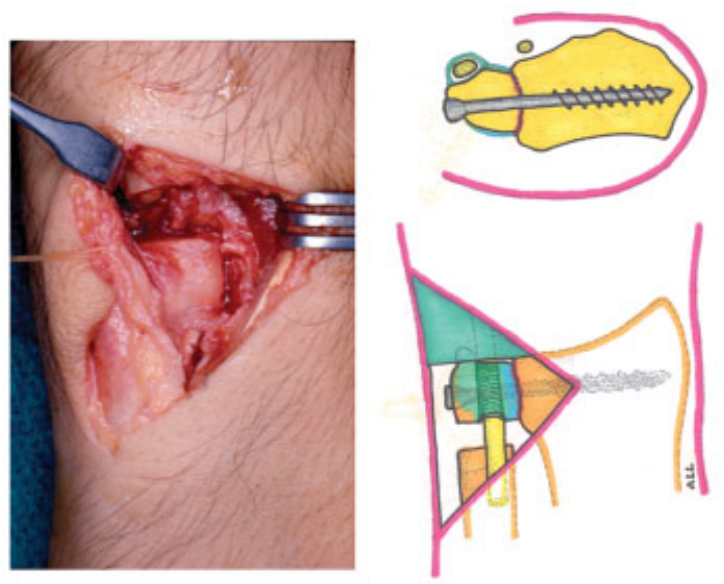

a

Fig. 6 (a) The screw is advanced until the bone surfaces to be arthrodesed have been moderately compressed and the head of the screw is introduced slightly into the head of the ulna. (b) The bone gap of the osteotomized ulna should measure $\sim 5 \mathrm{~mm}$, and the ECU tendon should be located at the dorsum. 
as this will cause unnecessary inflammation and pain at the site of the pseudoarthrosis, but should rotate the forearm until maximal pronation is achieved and maintain it in this position for a while. After resting it temporarily in neutral position, the patient should rotate the forearm in the opposite direction to obtain maximal supination.

Forearm rotation is better accomplished by passive mobilization with the elbow flexed $90^{\circ}$. Within a period of $\sim 3$ weeks the patient will achieve full pronation and supination of the forearm with minimal discomfort. However, during the first few weeks the patient may experience pain when carrying objects with the elbow flexed and the forearm in neutral position, as the weight of the object will displace the radius against the flexed ulna. The patient may also experience pain, with a feeling of bone snapping, when rotating the forearm from slight pronation to supination, and vice versa, from impingement of the stump of the osteotomized ulna against the ulnar border of the radius, particularly when carrying heavy objects.

\section{Results}

Radioulnar arthrodesis was obtained in all cases, since optimal stability and bone contact were provided by the compression lag screw.

Radiocarpal joint mobility was not altered by the procedure, and all patients regained full wrist joint mobility shortly after the procedure. Only the 10 patients in whom a corrective osteotomy of the distal radius was performed required a longer postoperative period to recover full joint mobility, mainly wrist flexion, due to postoperative fibrosis of dorsal capsular structures. However, the arch of extension and flexion was moved to a more flexed position, as the dorsal inclination of the distal radial joint was corrected by the osteotomy.

Full pronation and supination was regained in all patients, except for two who had minimal restriction of supination. Calcification at the osteotomy site was observed in four cases, three of whom had a simultaneous distal radial osteotomy performed. Only two out of these four patients required excision of the calcification because it caused restriction of forearm rotation. Three other patients complained of pain and presented inflammatory signs at the site of the pseudoarthrosis with intensity and duration longer than the average, which generally speaking was for about 3 weeks. Symptoms disappeared within an average of 3 months, after treatment with short periods of rest and antiinflammatory steroids.

When explored with the forearm in neutral position and the elbow flexed, all patients had increased passive displaceability of the proximal ulnar stump, although it was painless. However, when patients were holding objects or were asked to stabilize the wrist actively, the proximal ulna became stable from contraction of the PQ, FCU, and ECU muscles. At an average of 6.5 years after the procedure, none of the patients complained of painful instability throughout the entire range of pronation-supination while holding heavy objects with their hands. All patients returned to their previous occupations.

\section{Discussion}

In addition to the general complications related to any surgical procedures, only three complications can occur directly related to the S-K procedure: nonunion or delayed union of the arthrodesis, fibrous or osseous union at the pseudoarthrosis, and painful instability of the proximal ulna stump. The first two are not of much concern, as they can be easily addressed. However, a painful instability of the proximal ulna stump can cause a serious disability, which in most cases can be very difficult to correct. $^{12}$

Some authors recommend using a segment of the ulna that has been resected as a bone graft..$^{13-15}$ We do not recommend interposing a bone graft between the radius and ulna, as this creates an unnecessary barrier of devascularized tissue, particularly if cortical bone from the ulna is used. The use of a compression screw ensures the arthrodesis.

To prevent instability of the proximal ulnar stump, some authors recommend to perform a tenodesis with a slip of the ECU or FCU tendons preserving its distal attachment into the pisiform bone. ${ }^{16,17}$ In all of theses cases, the osteotomy was done too proximal, to stabilize the distal ulna with two screws, and often the PQ muscle was sacrificed for interposition at the pseudoarthrosis.

The best results will be obtained if the pseudoarthrosis is done at the level of the ulnar head, removing only $5 \mathrm{~mm}$ of bone. ${ }^{18,19}$ With the passing of time, the bone defect will get larger from resorption of the bone ends, mainly the proximal one (-Figs. 7a,b). Impingement of the proximal ulna will occur in a relatively flat, triangular-shaped surface, just proximal to the sigmoid notch joint. Further proximally, the ulnar surface of the radius becomes a ridge for the insertion of the IOM, causing painful snapping of the ulnar stump during pronation and supination of the forearm. The reason why a painful instability of the proximal ulna stump is more commonly observed after the S-K procedure as opposed to the Darrach is because the osteotomy of the ulna is done proximally in the Sauvé-Kapandji procedure.

The axis of rotation of the forearm runs obliquely from the center of the radial head proximally to the center of the ulnar
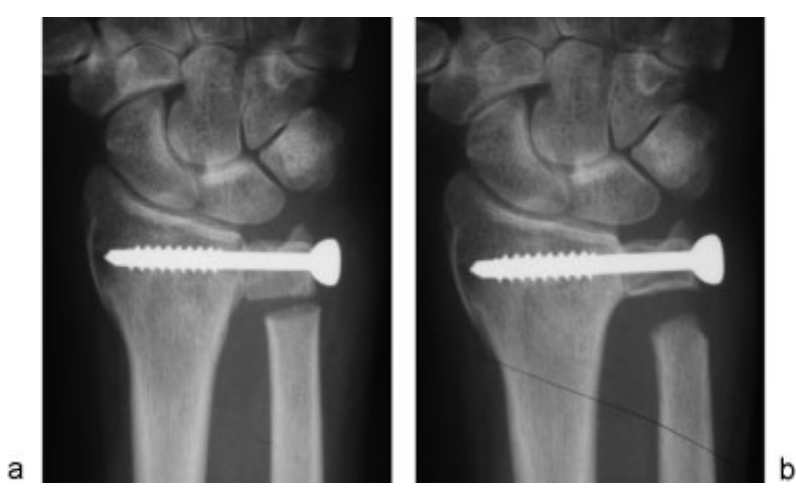

Fig. 7 (a) Radiograph of the wrist showing the head of the ulna compressed against the radius with a malleolar screw, with a very small segment of ulna removed. (b) With the passing of time, the bone defect will get larger from resorbtion of the bone ends, mainly the proximal ulna stump. 

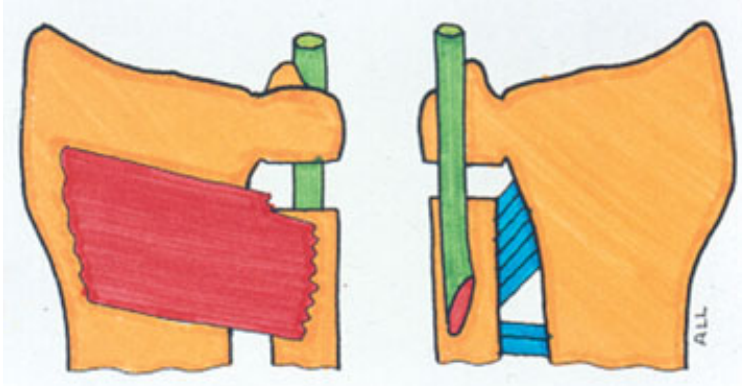

Fig. 8 Schematic drawing of the structures providing stabilization of the proximal ulna. On the left, an anterior view showing the pronator quadratus. The FCU is not shown. On the right, a posterior view showing the interosseous membrane and the extensor carpi ulnaris tendon.

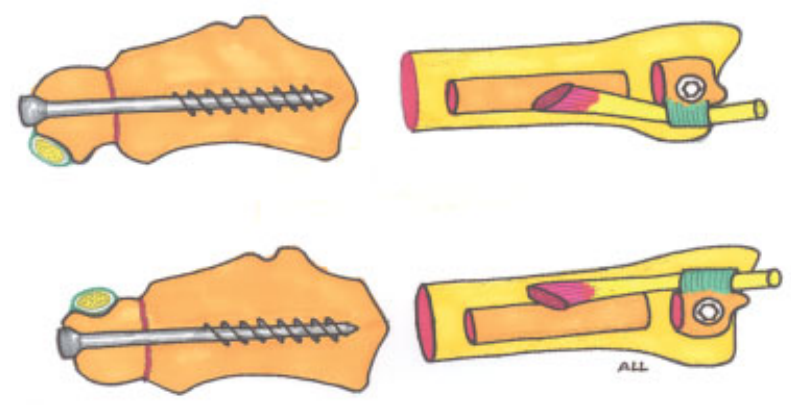

Fig. 9 With the forearm in pronation, the ECU tendon is located below the axis of rotation of the DRUJ. As the surgery is usually done with the forearm in this position, it is not uncommon to place the entrance of the screw dorsal to the ECU inadvertently, as shown in the top drawing. Using the technique here described, the surface of the ulna to be arthrodesed is the one facing the surgeon while the forearm is

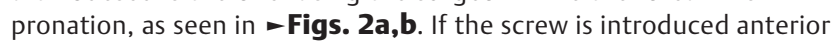
to the ECU tendon, the latter will be displaced dorsally for added stability of the proximal ulna, as depicted at the bottom drawing.

head distally. The pseudoarthrosis should be made as close as possible to the head of the ulna. An osteotomy at a more proximal level will cause a divergence of movements between the proximal ulna stump and the head of the ulna, which has already been fixed to the radius. Another reason for creating the most distal pseudoarthrosis as possible is so as not to disturb the static and dynamic structures that provide stability to the proximal ulna: the $\mathrm{PQ}, \mathrm{ECU}$, and FCU muscles and IOM insertions ${ }^{20-23}$ (-Fig. 8). The deep head of the PQ is the main dynamic stabilizer of the proximal ulna and should not be stripped of any length and used as interposition between both ends of the ulna. The stability of the DRUJ should be explored with the elbow flexed and the forearm in neutral position. In this position, some degree of anteroposterior displacement of the ulna will be observed, which will be increased in patients with joint hyperlaxity, mainly young women. This instability will not be observed if patients are simply asked to stabilize the ulna "dynamically." This is accomplished by contraction of the PQ muscle, demonstrating how important it is not to disinsert the PQ muscle from the ulna.
The ECU tendon, inside its fibro osseous tunnel, also plays an important role in the stabilization of the DRUJ. ${ }^{24}$ If the ulnar head has been arthrodesed to the distal radius, it does not need the ECU for stabilization, but it will still provide some stabilization to the proximal ulna stump. This will be more effective when the osteotomy is made very close to the ulnar head, the sheath is not disrupted from the ulna, and the ECU is placed dorsal to the osteotomy (-Fig. 9).

\section{Conflict of Interest \\ None}

\section{References}

1 Lichtman DM, Ganocy TK, Kim DC. The indications for and techniques and outcomes of ablative procedures of the distal ulna. The Darrach resection, hemiresection, matched resection, and Sauvé-Kapandji procedure. Hand Clin 1998;14(2):265-277

2 Malgaine JF. Traité des fractures et des luxations. Vol 2. Paris: JB Braillière; 1855

3 Darrach W. Anterior dislocation of the head of the ulna. Ann Surg 1912;56:802-803

4 Darrach W. Partial excision of lower shaft of the ulna for deformity following Colles's fracture. Ann Surg 1913;57:764-765

5 Baldwin WI. Orthopaedic surgery of the hand and wrist. In: Jones R, ed. Orthopaedic Surgery of Injuries. London, England: Henry Frowde and Hodder \& Stoughton; 1921:241-282

6 Sauvé L, Kapandji M. Nouvelle technique de traitement chirurgical des luxations récidivantes isolées de l'extrémité inférieure du cubitus. J Chir (Paris) 1936;47:589-594

7 Berry JA. Chronic subluxation of the distal radio-ulnar articulation. Br J Surg 1931;18:526-527

8 Petersen MS, Adams BD. Biomechanical evaluation of distal radioulnar reconstructions. J Hand Surg Am 1993;18(2): 328-334

9 Alnot JY, Faroux L. La synovectomie réaxation stabilisation du poignet rhumatoïde incluant l'opération de Sauvé Kapandji. In: Tubiana R, ed. Traité de chirurgie de la main. Vol. 5. Paris, France: Masson; 1995:442-452

10 Obry C, Tran Van F, Fardellon P, Marrasse E, Decoopman M. L'association synovectomie postérieure, réaxation carpienne et opération de Sauvé Kapandji dans le traitement du poignet rhumatoïde. A propos de 60 cas. La Main 1996;1:299-306

11 Garcia-Elias M. Radioulnar instability. Current Orthopaedics 1999;13:283-289

12 Lees VC, Scheker LR. The radiological demonstration of dynamic ulnar impingement. J Hand Surg [Br] 1997;22B:448-450

13 Kapandji IA. The Kapandji-Sauvé operation. Its techniques and indications in non rheumatoid diseases. Ann Chir Main 1986;5 (3):181-193

14 Minami A, Suzuki K, Suenaga N, Ishikawa J. The Sauvé-Kapandji procedure for osteoarthritis of the distal radioulnar joint. J Hand Surg Am 1995;20(4):602-608

15 Nakamura R, Tsunoda K, Watanabe K, Horii E, Miura T. The SauvéKapandji procedure for chronic dislocation of the distal radioulnar joint with destruction of the articular surface. J Hand Surg [Br] 1992;17(2):127-132

16 Breen TF, Jupiter JB. Extensor carpi ulnaris and flexor carpi ulnaris tenodesis of the unstable distal ulna. J Hand Surg Am 1989;14 (4):612-617

17 Lamey DM, Fernandez DL. Results of the modified Sauvé-Kapandji procedure in the treatment of chronic posttraumatic derangement of the distal radioulnar joint. J Bone Joint Surg Am 1998;80 (12):1758-1769 
18 Lluch A, Garcia-Elias M. The Sauvé-Kapandji procedure: technical considerations. Orthop Surg Techniques 1995;9:67-70

19 Lluch A. The Sauvé-Kapandji procedure: indications and tips for surgical success. Hand Clin 2010;26(4):559-572

20 Gabl M, Zimmermann R, Angermann P, et al. The interosseous membrane and its influence on the distal radioulnar joint. An anatomical investigation of the distal tract. J Hand Surg $[\mathrm{Br}]$ 1998;23(2):179-182

21 Johnson RK, Shrewsbury MM. The pronator quadratus in motions and in stabilization of the radius and ulna at the distal radioulnar joint. J Hand Surg Am 1976;1(3):205-209
22 Stuart PR. Pronator quadratus revisited. J Hand Surg [Br] 1996;21 (6):714-722

23 Ward LD, Ambrose CG, Masson MV, Levaro F. The role of the distal radioulnar ligaments, interosseous membrane, and joint capsule in distal radioulnar joint capsule in distal radioulnar joint stability. J Hand Surg [A] 2000;25A:341-351

24 Spinner M, Kaplan EB. Extensor carpi ulnaris. Its relationship to the stability of the distal radio-ulnar joint. Clin Orthop Relat Res 1970;68:124-129 